\title{
Adaptive Optics pre-compensation for GEO feeder links: the FEEDELIO experiment
}

A. Montmerle Bonnefois, J-M. Conan, C. Petit, C. B. Lim, S. Meimon, J-F. Sauvage, V. Michau, P. Perrault, F. Mendez, B. Fleury, J. Montri, N. Védrenne aurelie.bonnefois@onera.fr

Special thanks to Z. Sodnik, C. Volland and C. Heese from ESA ONERA

THE FRENCH AEROSPACE LAB 


\section{Limit to AO performance : the angular decorrelation}

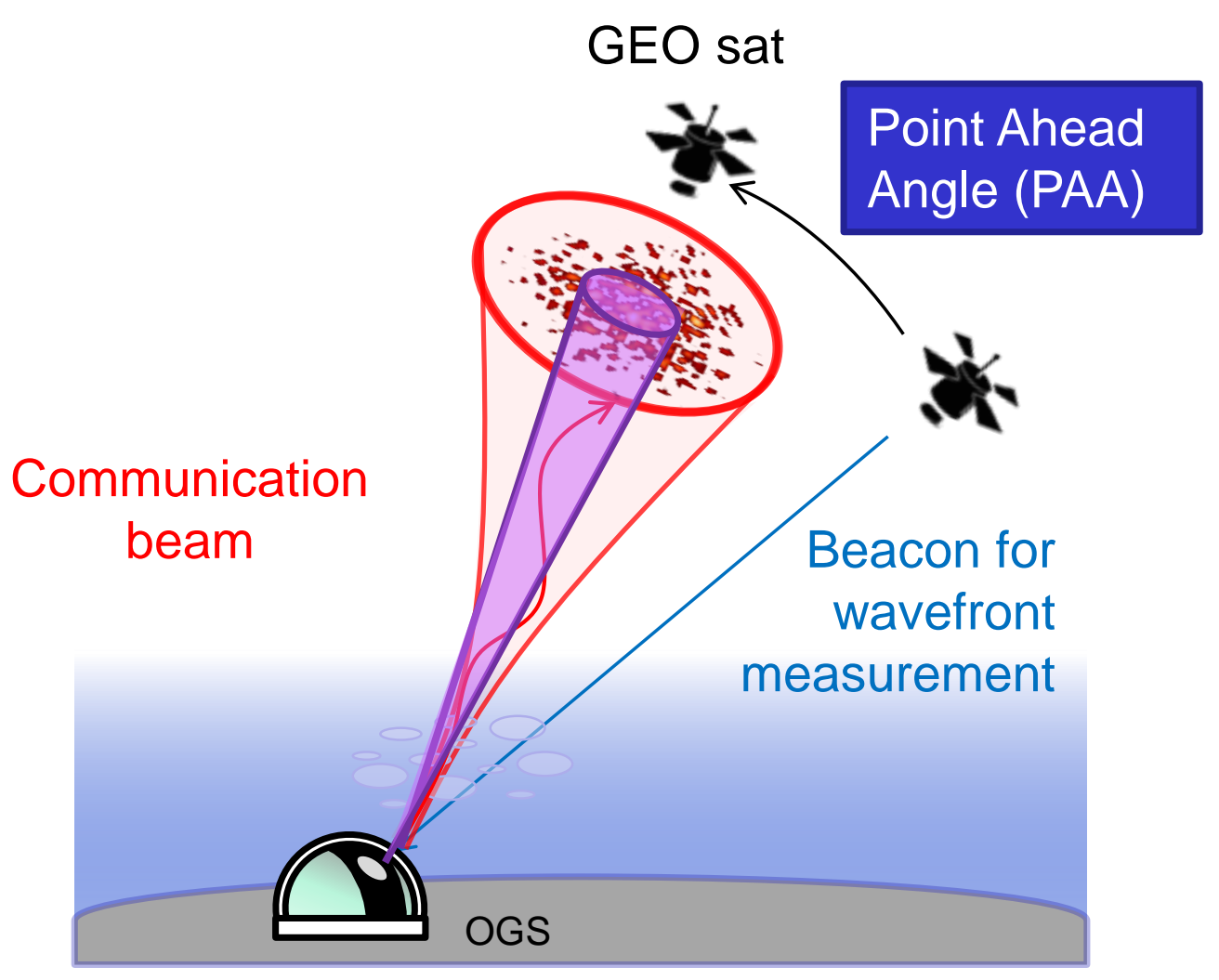

PAA $18 \mu \mathrm{rad}$

$\rightarrow$ Same order as atm. turbulence statistics

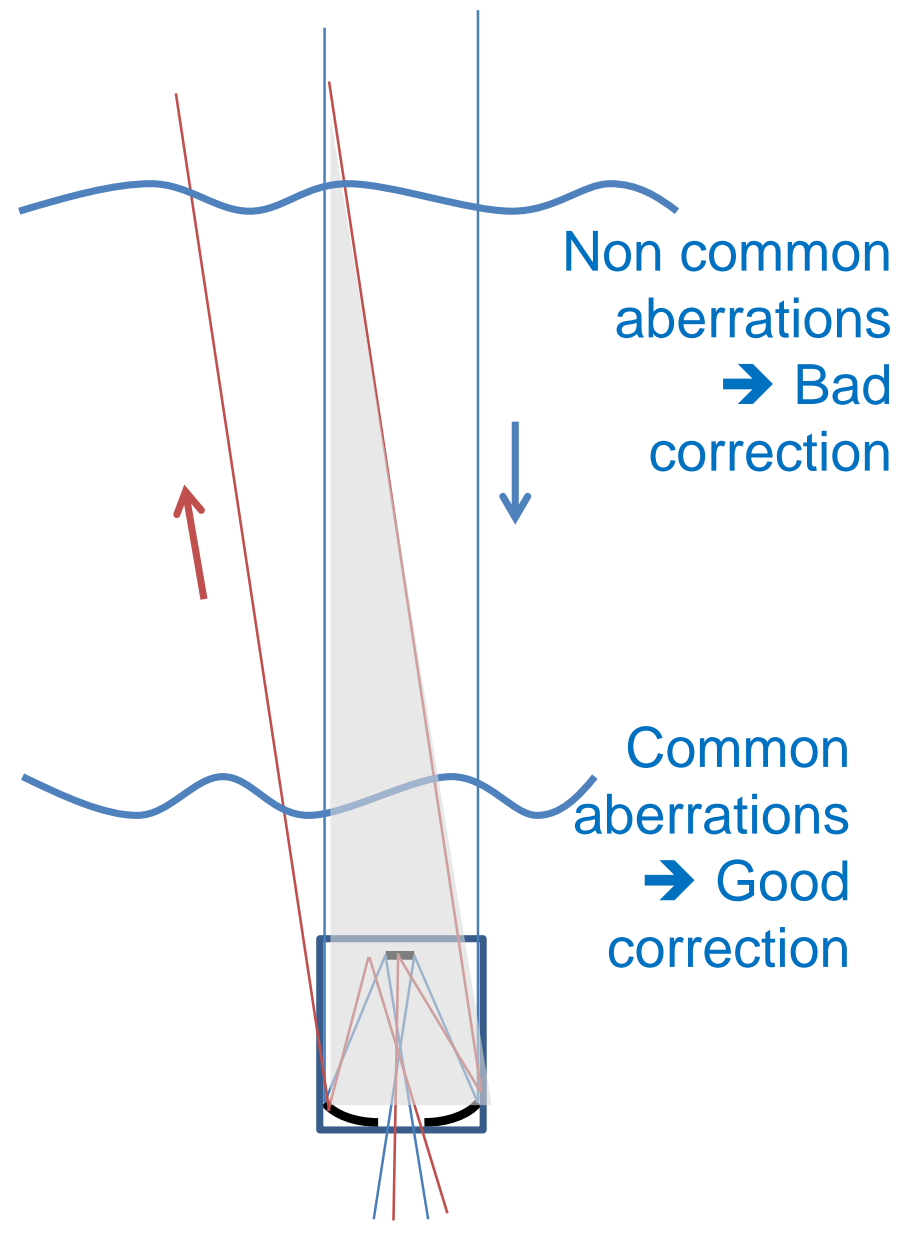

$\rightarrow$ AO Perf. depends on turb. profile $\rightarrow$ Angular decorrelation 


\section{Goals of the FEEDELIO experiment}

\section{$\rightarrow$ FEEDELIO experiment (FEEDEr LInk with adaptive Optics)}

- Evaluating the potential and limitation of AO for GEO-Feeder Links regarding angular decorrelation,

$\checkmark$ Experimental demonstration + comparison to performance derived from inhouse existing models

What we want to measure :

> Signal statistics: average optical power and optical power fluctuations

$>$ with PAA (for angular decorrelation) and without PAA (for reference)

$>$ Without $\mathrm{AO}$, with Tipt-tilt only, and up to 5 radial orders $\mathrm{AO}$ correction

$\square$ Not possible with a GEO satellite $\rightarrow$ slant path experiment in Teide 


\section{FEEDELIO slant path demonstration of a GEO Feeder Link}
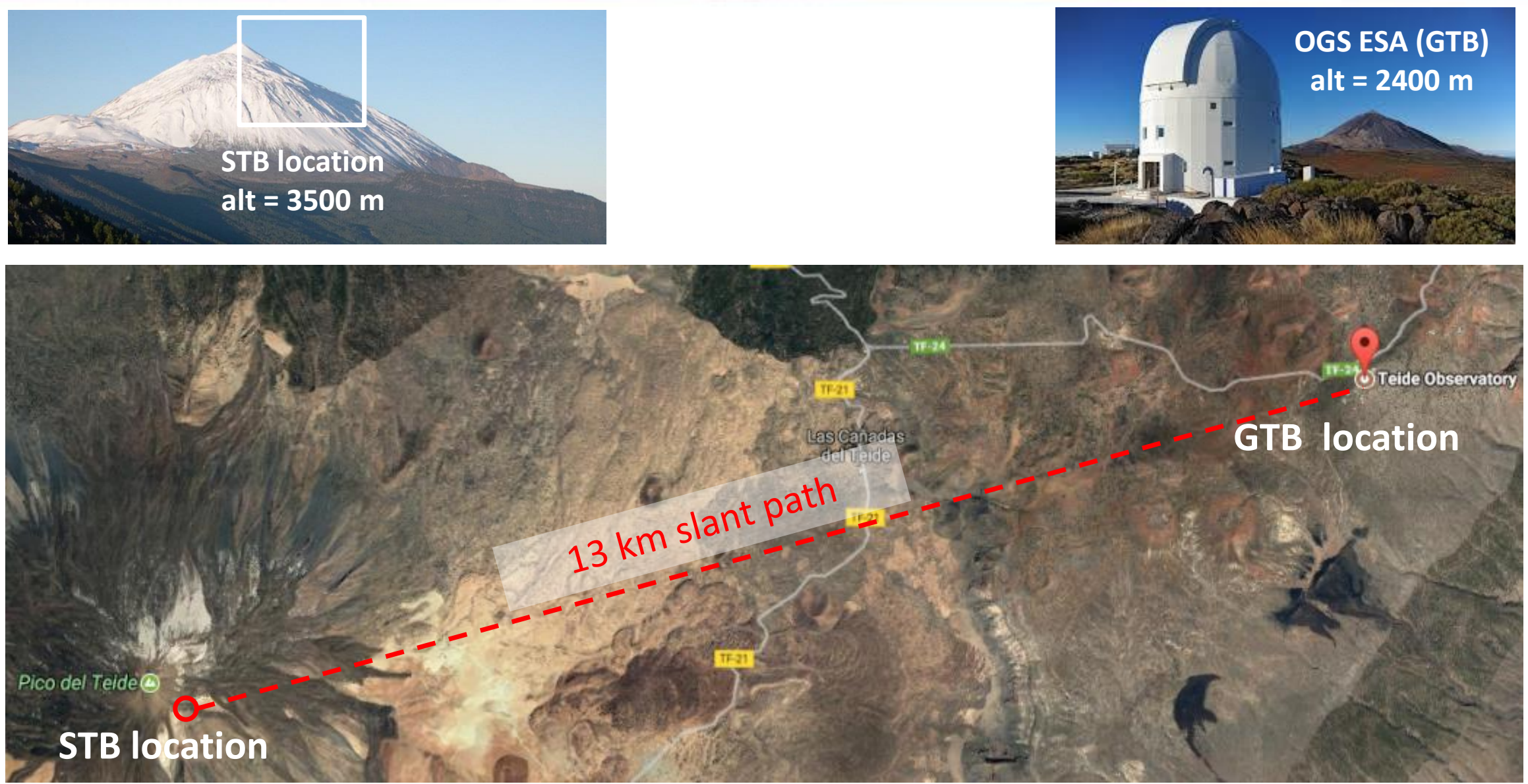

STB : Satellite Terminal Breadboard GTB : Ground Station Terminal Breadboard Angular decorrelation similar to a GEO Feeder Link 


\section{A closer look on Satellite Terminal Breadboard (STB)}

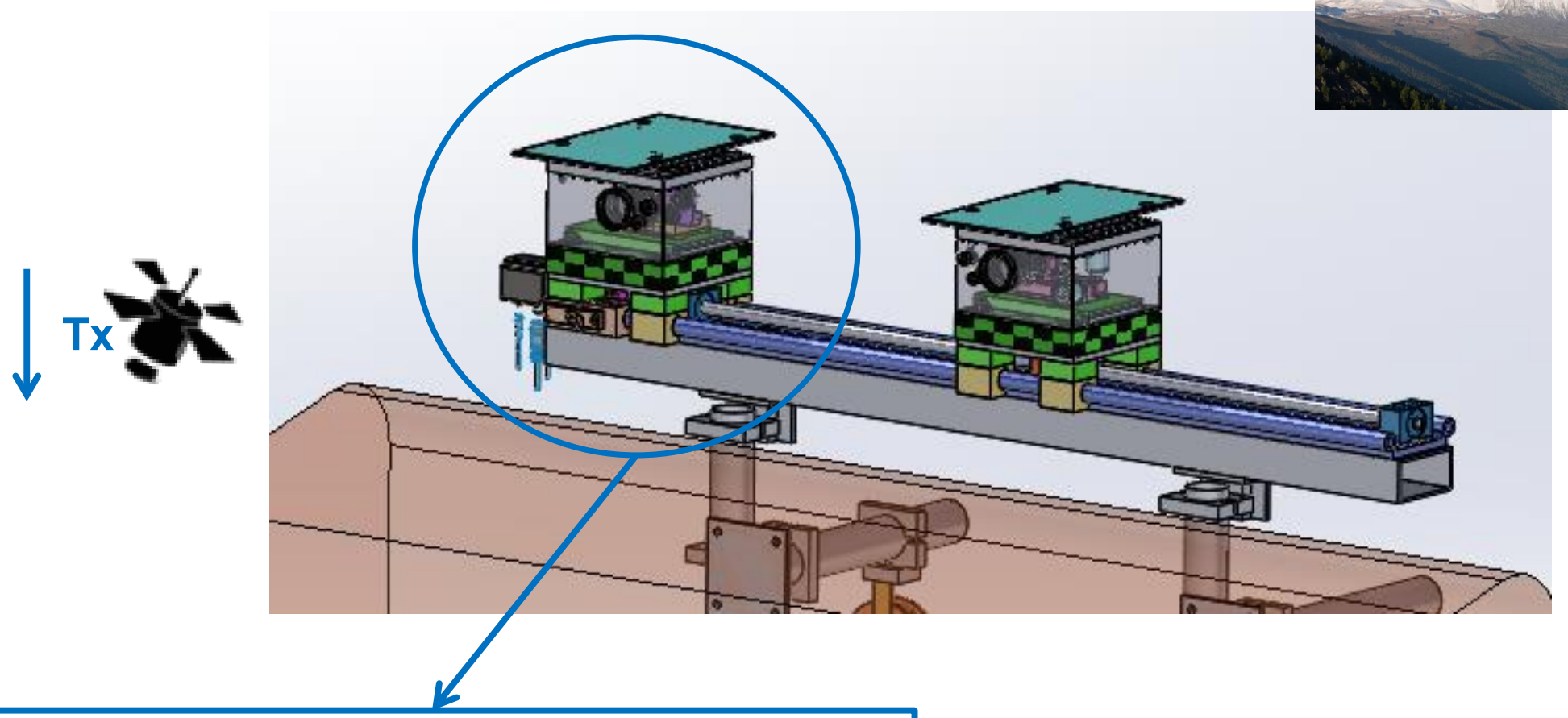

\section{On-axis module:}

$>=$ GEO Satellite Transmitter emulator

$>$ Emits downlink ref beam for $\mathrm{AO}$

+ Reference for uplink signal statistics $(\mathrm{PAA}=0)$ 


\section{A closer look on Satellite Terminal Breadboard (STB)}

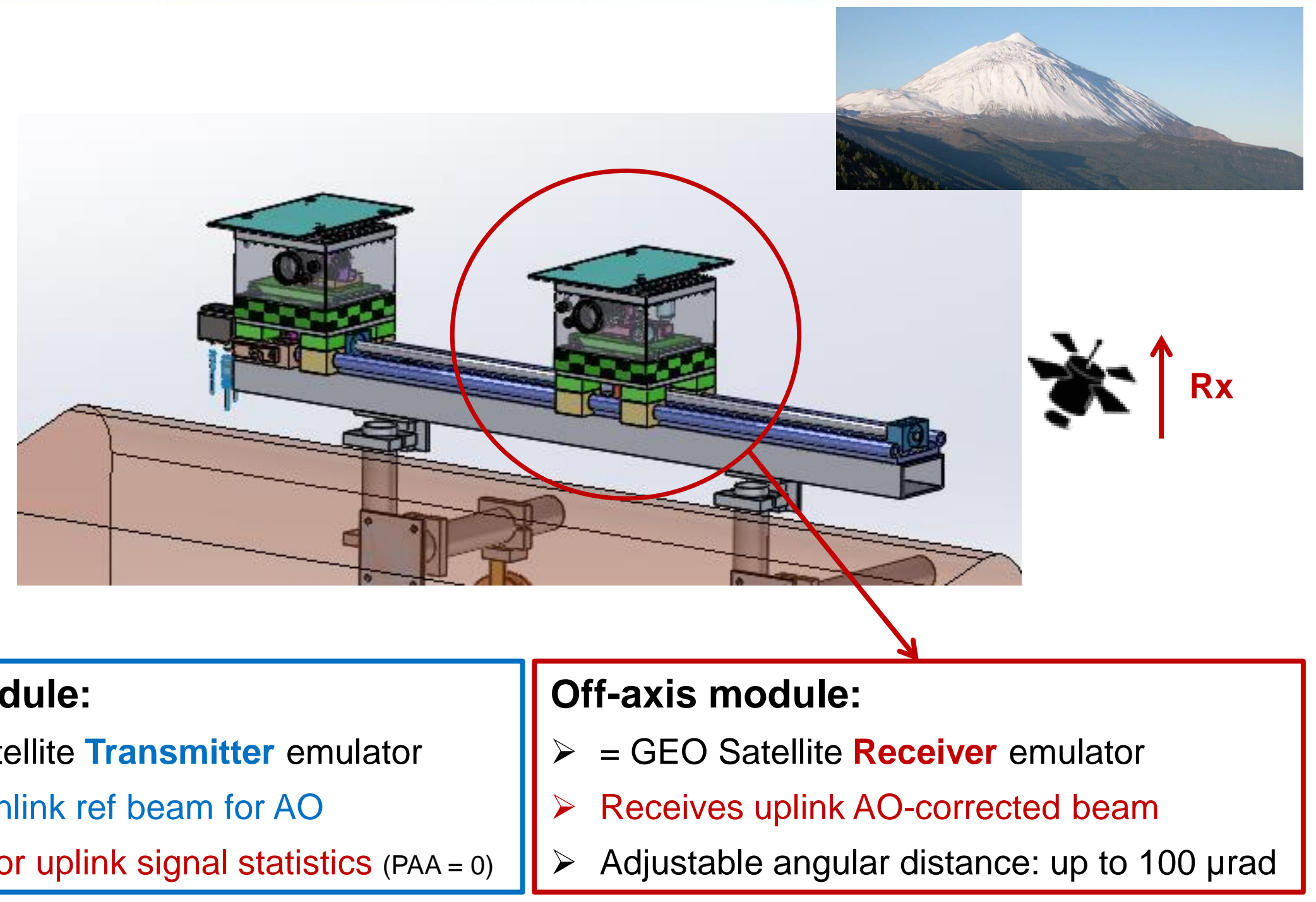




\section{A closer look on Ground Terminal Breadboard (GTB)}

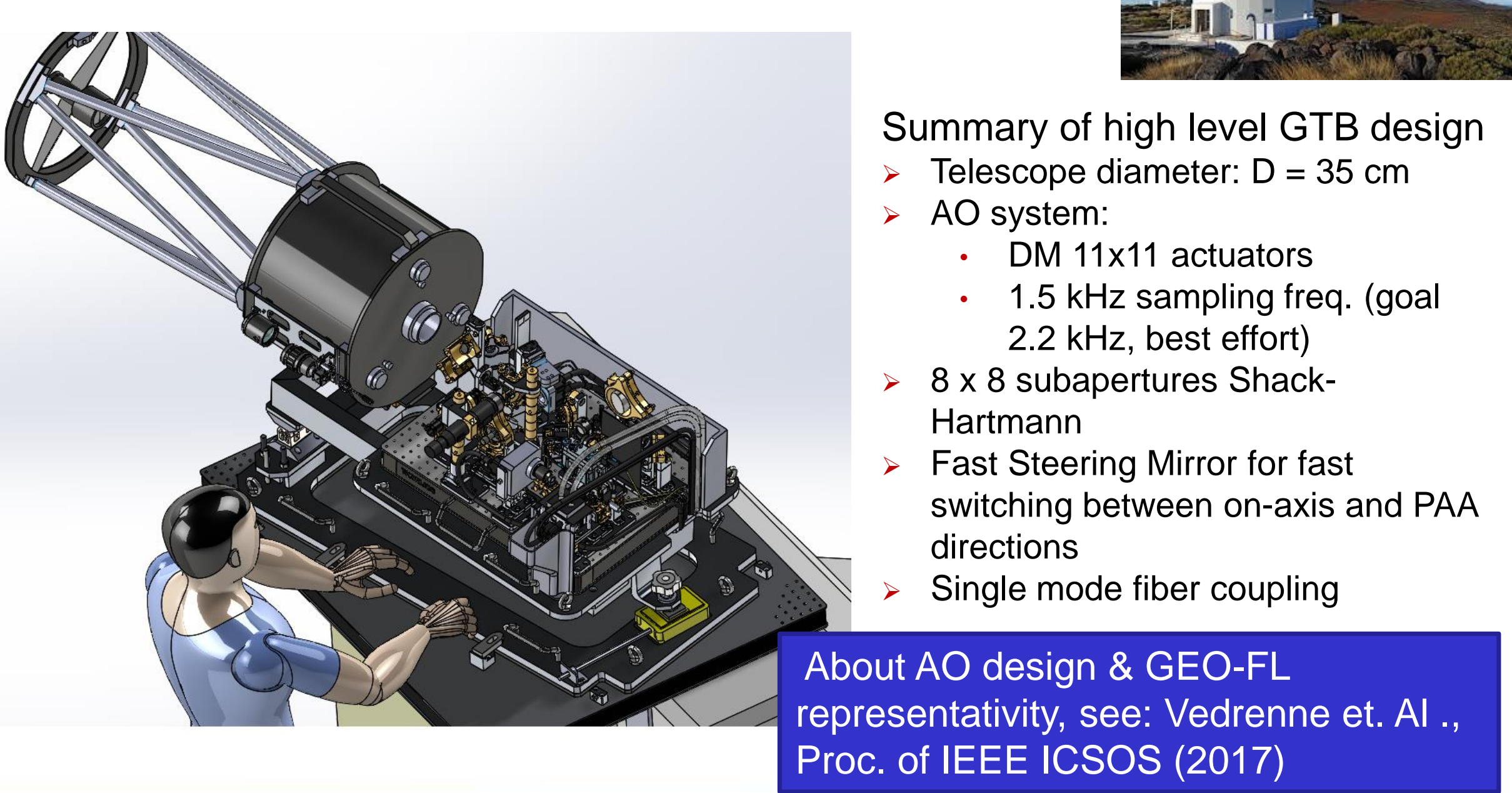




\section{From principle...}

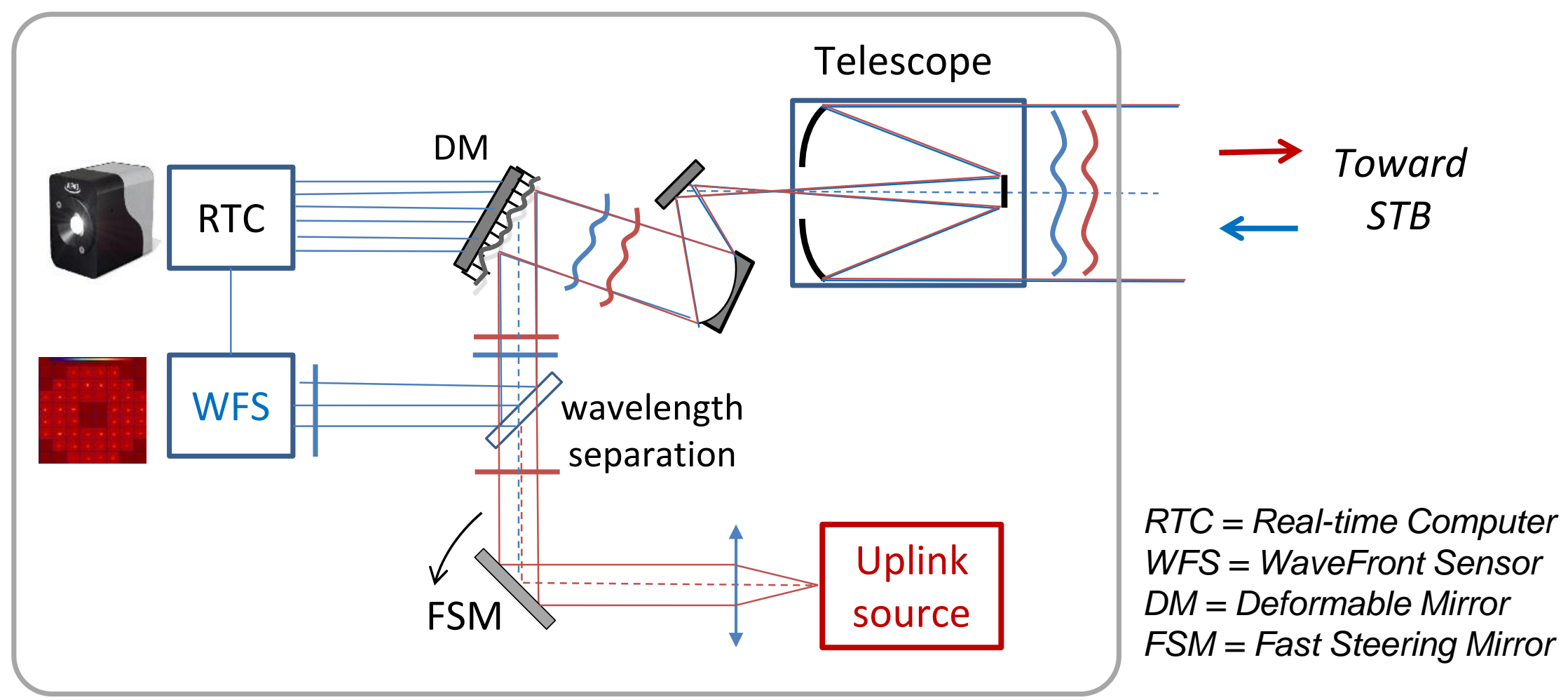

\section{GTB:}

$>$ Analyses turbulence on downlink beam

> Pre-compensates turbulence on uplink beam

$>$ Pointing Angle controled through FSM $(\rightarrow$ PAA) 


\section{From principle... to final architecture}

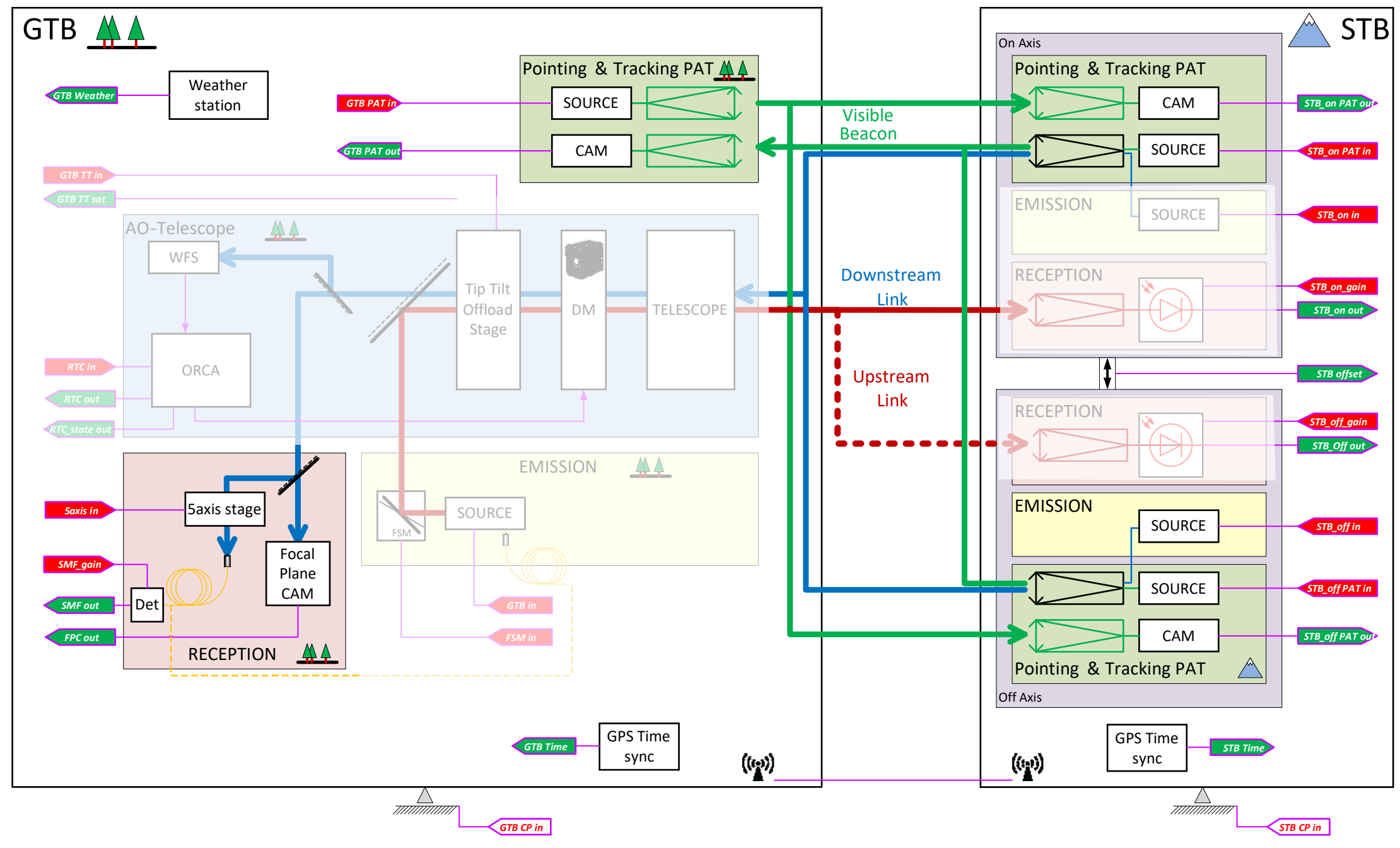

$\rightarrow$ Several tools for alignment, calibration \& monitoring 


\section{Controlling the measurements: AO monitoring}

- Recorded data for AO monitoring

$>$ real-time diagnostics tools:

- Focal plane control camera

- AO loop residuals

- Single mode fiber downlink injection efficiency

$>$ optical power collected on the uplink

- for both on an off axis,

- with variable number of corrected modes

$\rightarrow$ AO error budget for angular decorrelation effect quantification 


\section{Controlling the measurements: PAA and turbulence monitoring}

Average power loss for GEO and FEEDELIO scenarios (power fluctuations = idem)

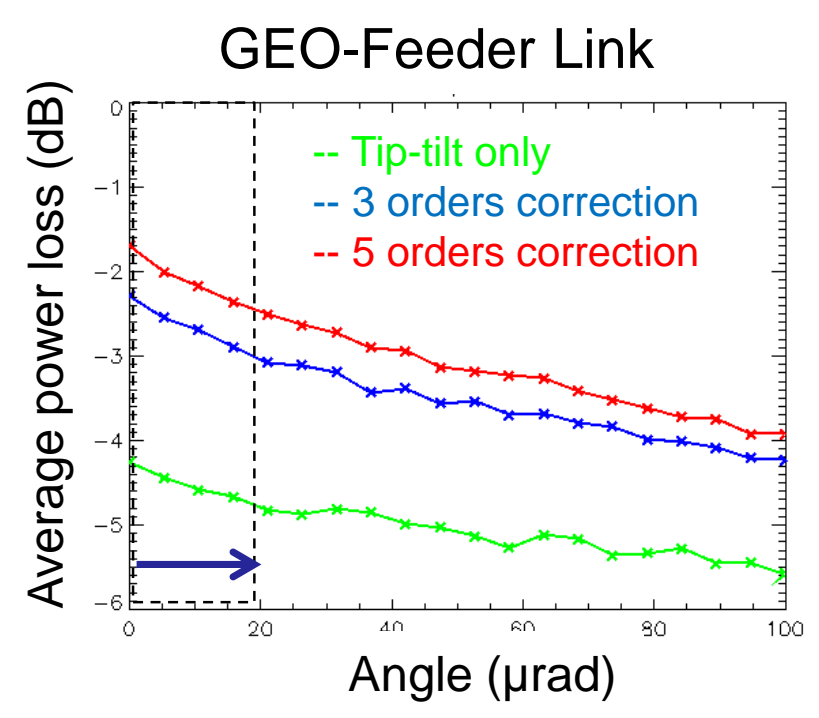




\section{Controlling the measurements: PAA and turbulence monitoring}

Average power loss for GEO and FEEDELIO scenarios (power fluctuations = idem)

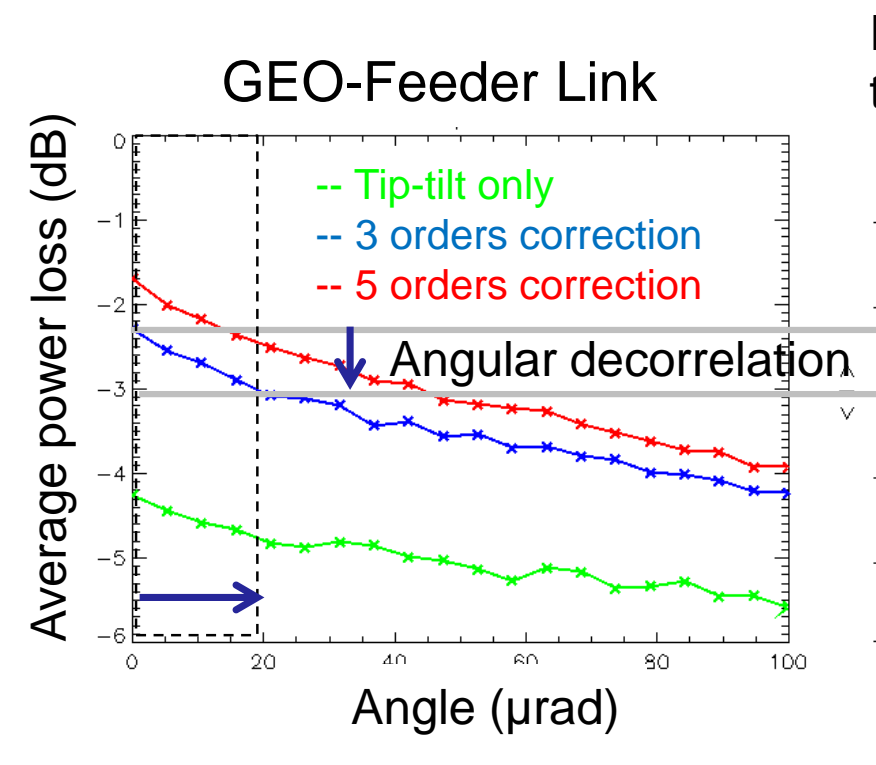

FEEDELIO, Stable Monin Obukov turbulence profile

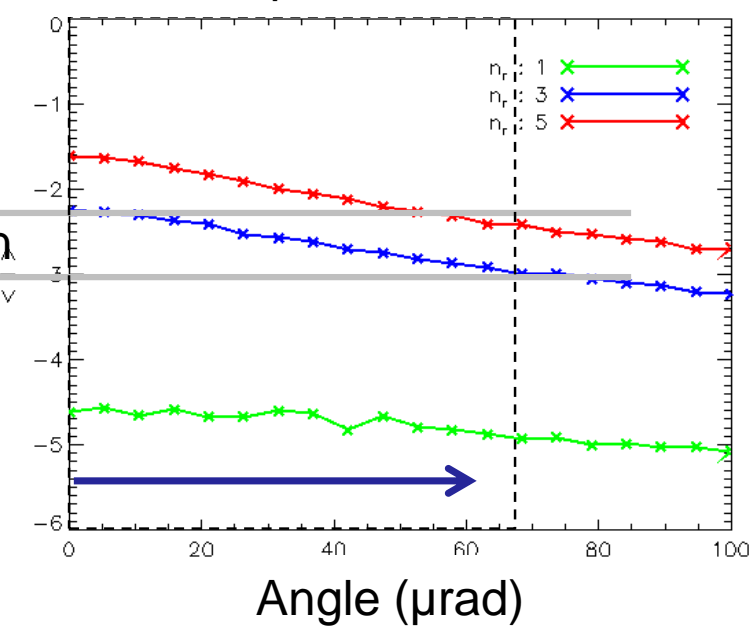

FEEDELIO, Strong unstable Monin Obukov turbulence profile

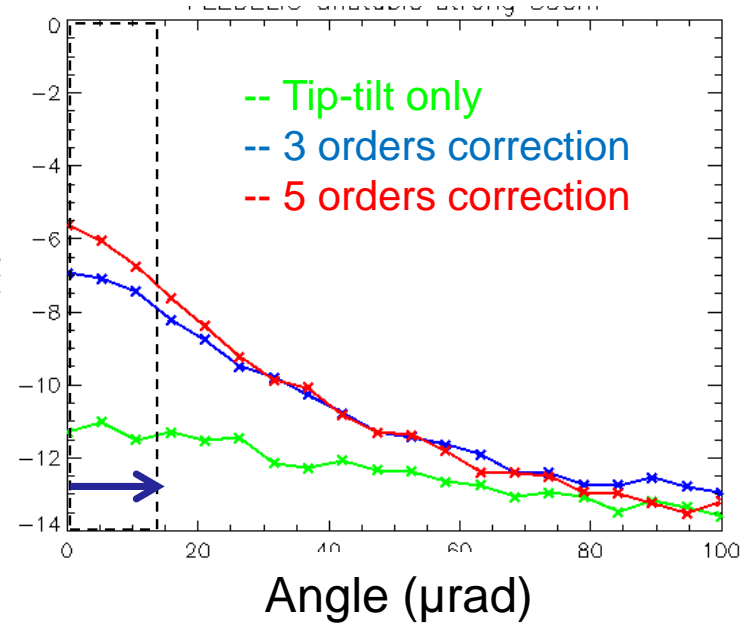

Equivalent PAA evolves with turbulence conditions ( $r_{0}$ typ. evolution $10 \mathrm{~min}$ )

$>$ Motorized off-axis STB module

> «Fast » switching between the modules with FSM at GTB

$\rightarrow$ Sequence of alternate measurements / on axis, off-axis / $\mathrm{nr}=1,3,5$

$\rightarrow$ Turbulence profile estimated from WFS measurements 


\section{Current status? integration is ongoing...}

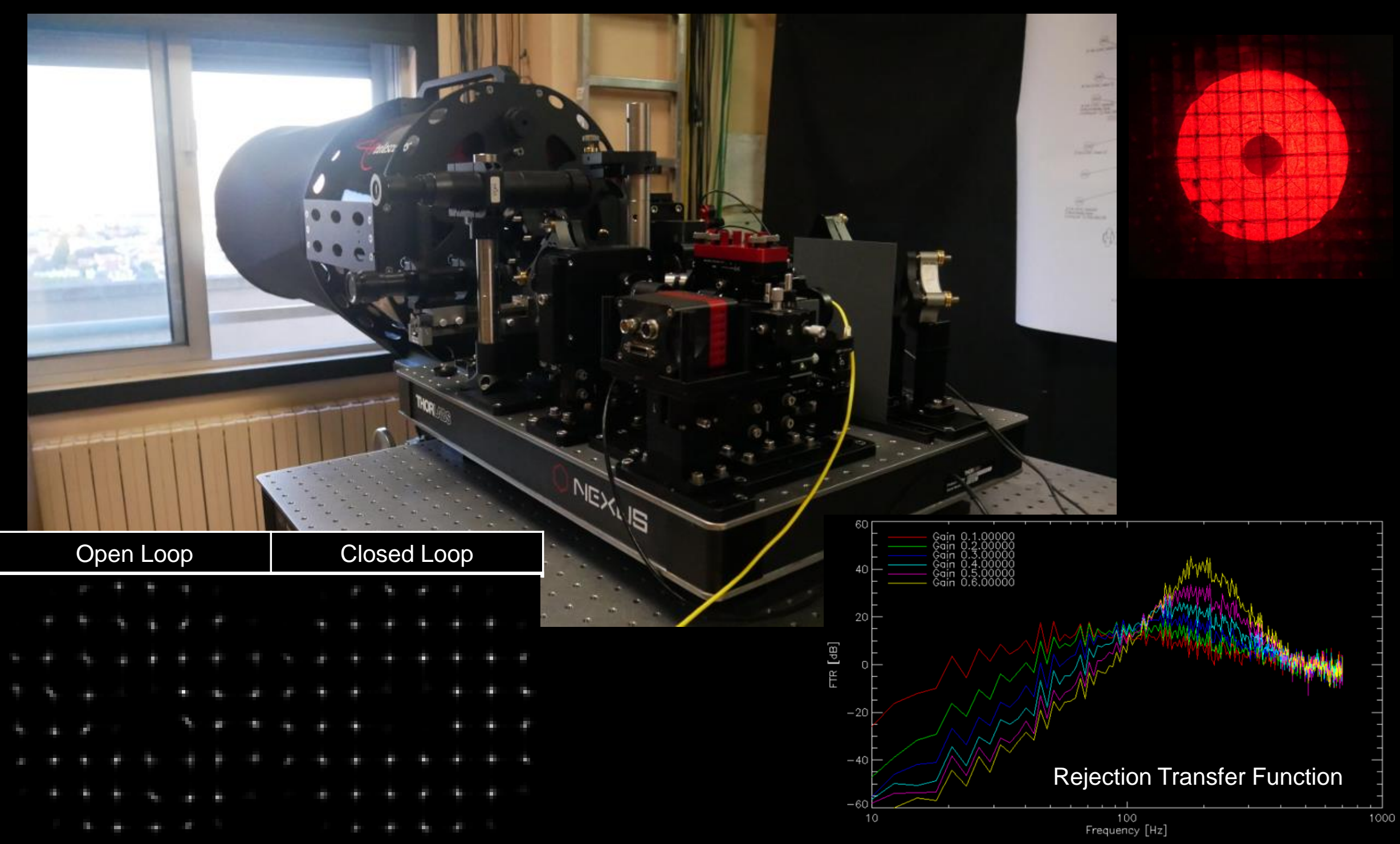




\section{Conclusion}

- Experiment critical for the validation of PAA effect on AO correction

$\square$ Careful design for accurate and detailed data analysis

$\checkmark$ Ongoing integration of the breadboards

\section{Coming next :}

- Overall functional tests planned at the end of $2018(4 \mathrm{~km}$ horizontal path)

- Final experiment in representative environment planned in spring 2019
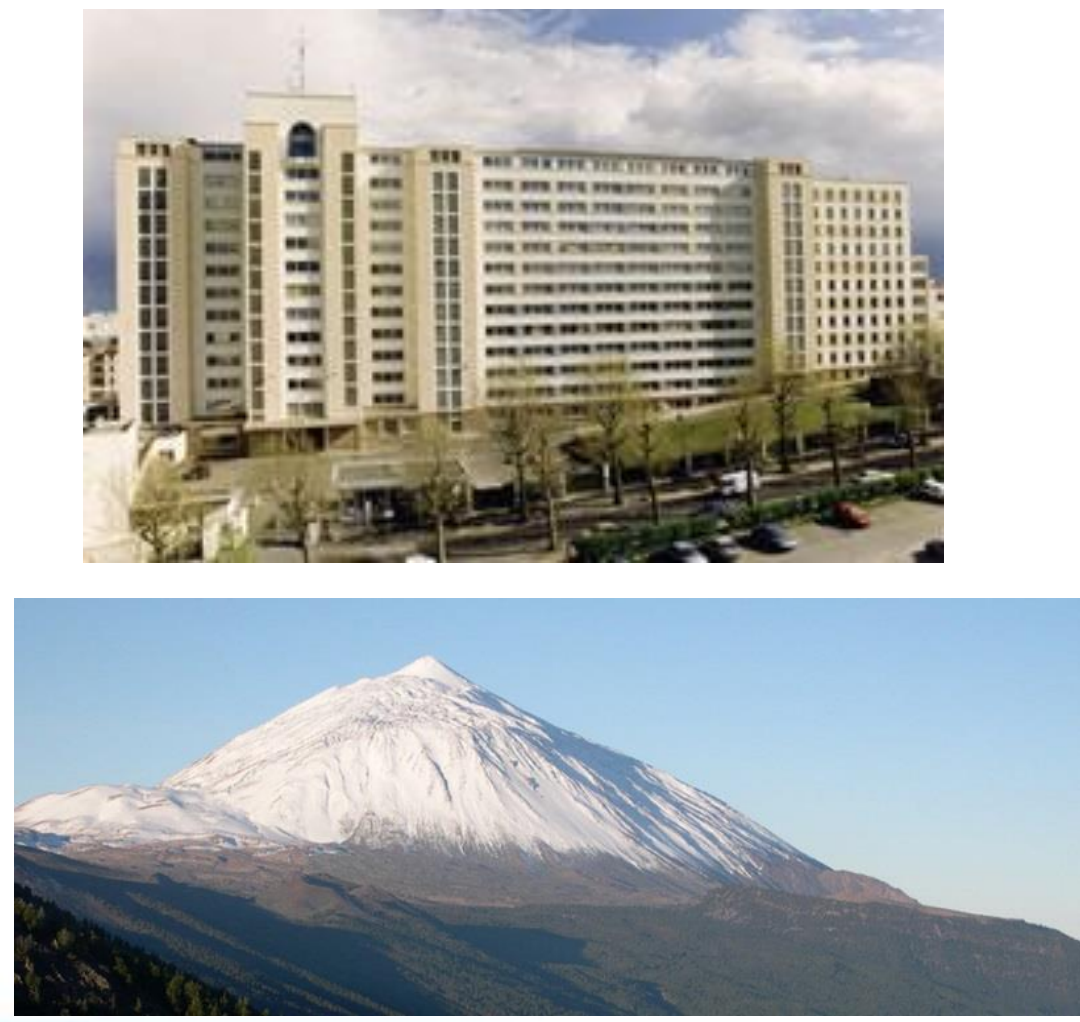